\title{
LA FORMACIÓN INICIAL DOCENTE DESDE LA RESPONSABILIDAD SOCIAL UNIVERSITARIA: SATISFACCIÓN DEL ALUMNADO EN RELACIÓN A UNA EXPERIENCIA DE APRENDIZAJE-SERVICIO
}

\section{INITIAL TEACHER TRAINING FROM UNIVERSITY SOCIAL RESPONSIBILITY: STUDENT SATISFACTION ON A SERVICE-LEARNING EXPERIENCE}

\author{
Ma José Mayorga-Fernández \\ mjmayorga@uma.es \\ Dolores Madrid-Vivar \\ Imadrid@uma.es \\ Lourdes De La Rosa Moreno \\ Idelarosa@uma.es \\ Facultad de Ciencias de la Educación. Universidad de Málaga (España)
}

Recibido: 06/04/2021

Aceptado: 20/06/2021

\begin{abstract}
Resumen:
El objetivo de este artículo es conocer el nivel de satisfacción de los estudiantes en una experiencia de Aprendizaje-Servicio (ApS) desarrollada en la Facultad de Ciencias de la Educación de la Universidad de Málaga. La muestra ha sido de 76 estudiantes, de 2 grupos diferentes, del Grado de Maestro en Educación Infantil. Se planteó una investigación diagnóstico-descriptiva, ex post-facto, desde la complementariedad metodológica cualitativacuantitativa, para comprender las reflexiones y valoraciones del alumnado en relación a su experiencia de ApS. Los resultados muestran un nivel de satisfacción del alumnado muy elevado, aunque con diferencias significativas entre los Grupos A y B. Los ítems más valorados han sido la capacidad de trabajo en equipo, el fomento de la creatividad y la implicación en el servicio. Se concluye que es necesario aumentar el uso de esta estrategia metodología para potenciar el desarrollo de la Responsabilidad Social Universitaria.
\end{abstract}

Palabras clave: Responsabilidad Social Universitaria, Aprendizaje-Servicio, formación inicial profesorado, relación escuela-comunidad, metodologías didácticas 


\section{Abstract:}

The aim of this article is to know the level of satisfaction of students in a learning experience Service (APS) developed at the Faculty of Education at the University of Málaga. The experience included 76 Early Education Degree students divided into two different groups (A and B groups). The study is diagnostic-descriptive and ex-post facto which includes the complementary qualitative-quantitative evaluation methodology. Its main goals are to give an insight on the ratings and personal evaluation of such students after their Service Learning experiences. Results show highly positive ratings, with some quite significant differences between groups $\mathrm{A}$ and B. Highest values were linked to the following items: team work, promotion of creativity and personal implication into Service Learning activities. It is concluded that it is necessary to increase the use of this strategy methodology to enhance the development of University Social Responsibility.

Key-words: University social responsibility, Service-learning, Initial teacher training, schoolcommunity relationship, teaching methodologies

\section{Introducción}

Desde hace más de dos décadas, el neoliberalismo es la política que está impregnado la cultura social, así como la cultura política de las élites gobernantes (Angulo, 1995). Dicha política defiende lo económico por encima de lo político y social. Esto afecta sobremanera al sistema educativo, puesto que se prioriza la rentabilidad y eficacia, como si de una empresa se tratara. Ante este deplorable panorama socio-educativo, resulta prioritario que la Universidad sea capaz de formar ciudadanos competentes laboralmente, además de personas críticas, responsables y participativos en temas sociales (Santos, 2016), los cuales faciliten el cambio necesario del desarrollo político, económico y social del país. Para ello, es crucial una enseñanza universitaria de calidad, basada en la implicación, el entusiasmo, el compromiso y la crítica del alumnado, lo que lleva implícito una Responsabilidad Social Universitaria, cuestiones que ya están recogidas en la exposición de motivos de la LOU 6/2001, de 21 de diciembre. Dicha responsabilidad se debe entender como una política de mejora continua de la universidad hacia el cumplimiento efectivo de su misión social, mediante cuatro procesos: gestión ética y ambiental de la institución; formación de ciudadanos responsables y solidarios; producción y difusión de conocimientos socialmente pertinentes, así como, participación social en la promoción de un desarrollo más humano y sostenible (Vallaeys, 2008; Opazo et al., 2015). Las instituciones de Educación Superior, por tanto, deben tener como objetivo contribuir a la transformación de una sociedad más justa, poner fin a la pobreza, eliminar desigualdades y contrarrestar el cambio climático, de acuerdo a lo que se establece en la Agenda 2030 mediante los objetivos de Desarrollo Sostenible (ONU, 2019).

Desde este posicionamiento, la Universidad debe replantearse su misión y eficacia pedagógica (Zabalza, 2006) para generar impactos sociales y transformar la educación, contribuyendo a la formación de una ciudadanía más cívica (Jouannet et al., 2013; Simó, 2013; Lozano et al., 2018), es decir, la Universidad debe conseguir que su función educativa sea más eficaz (Martínez et al., 2013; Rizvi, 2010; Rodicio, 2010; Santos, 2016; Mayor y Rodríguez, 2017). La mejor forma de hacerlo es mediante el desarrollo y puesta en práctica de estrategias metodológicas que sean activas, participativas y con impacto social (Igelmo y Quiroga, 2018; Conde et al., 2019), como por ejemplo el Aprendizaje-Servicio (en adelante, ApS), donde tiene cabida la función ética de la Universidad, contemplando todas sus dimensiones: deontológica, ciudadana, cívica, humana, personal y social (Martínez, 2006). Esta idea queda reforzada por el Real Decreto 96/2014 de 14 de febrero, por el que se modifican los Reales Decretos 1027/2011, de 15 de julio, que establece 
el Marco Español de Cualificaciones para la Educación Superior (MECES), y 1393/2007, de 29 de octubre, que instaura la ordenación de las enseñanzas universitarias oficiales, donde se pone el énfasis en que los egresados universitarios sean capaces de realizar reflexiones éticas, independientemente del campo en el que se hayan formado, para poder contribuir a crear una sociedad más justa (Unesco, 2015). En este sentido, concretamente en España, ha sido la Conferencia de Rectores de las Universidades Españolas (CRUE, 2015, 2017) la encargada de promover acciones que promuevan el desarrollo de competencias y contenidos mediante estrategias metodológicas como el ApS, puesto que son consideradas herramientas muy potentes para el desarrollo de competencias personales y profesionales, así como para promover un aprendizaje ético. Dicha conferencia emitió un documento técnico aprobado por el Comité Ejecutivo y el Plenario de la Comisión de Sostenibilidad (Grupo CADEP), en el que insta a la institucionalización del ApS como estrategia docente en el marco de la Responsabilidad Social Universitaria (Mayor, 2020).

A este respecto, el artículo 64 del Real Decreto 1791/2010, de 30 de diciembre reconoce como derecho y deber del estudiante universitario "participar en las actividades formativas diseñadas para un correcto desarrollo de las actividades de participación social y cooperación al desarrollo, en las que solicite colaborar" (p. 109378) y la Ley 45/2015, de 14 de octubre, reconoce como uno de los ámbitos de actuación el voluntariado educativo entendido como:

acción solidaria planificada e integrada en el sistema y la comunidad educativa mejore las posibilidades de realización de actividades extraescolares y complementarias contribuyendo, en particular, a compensar las desigualdades que pudieran existir entre los alumnos por diferencias sociales, personales o económicas, mediante la utilización, entre otros, de programas de aprendizaje-servicio. (p. 95770-95771).

El ApS, por tanto, constituye una metodología idónea para la educación de una ciudadanía participativa, reflexiva y crítica capaz de contribuir a la búsqueda y construcción del bien común (Zayas et al., 2019). Se puede considerar como el desarrollo socioeducativo y solidario de la metodología de proyectos, donde tanto los estudiantes, como los demás agentes implicados, aprenden realizando un servicio a la comunidad, que conlleva un descubrimiento apasionado del estar, el hacer y el ser con sentido público en educación. Es decir, implica la puesta en práctica de proyectos innovadores que ejerzan un impacto positivo en la realidad social (Aramburuzabala et al, 2015; García y Lorente, 2014; Santos et al., 2017). El ApS constituye, en consecuencia, una estrategia metodológica articulada a través de prácticas pedagógicas y sociales que se apoyan en la reciprocidad, con sentido e impacto formativo y transformador, en la adquisición de conocimientos y competencias para la vida, en una pedagogía activa y reflexiva. Así como, en la construcción de una red de instancias de conexión y apoyo, donde se desarrollan competencias genéricas y específicas tanto para el buen desempeño en el mundo laboral (Martínez-Vivot y Folgueiras, 2015), como para el ejercicio de una ciudadanía participativa y solidaria. Se puede afirmar, por tanto, que consiste en toda una filosofía de cómo entender la Educación, en general, y la formación inicial del profesorado, en particular. Esta manera de entender dicha metodología conlleva una institucionalización curricular del ApS (García y Cotrina, 2015), es decir, un proceso de contaminación del currículo universitario, siguiendo un modelo abajo-arriba, asentado en la innovación educativa, facilitado desde la propia institución universitaria, todo un compromiso académico, moral, social y político. Mirar el ApS desde la dimensión institucional implica hacerlo desde la Responsabilidad Social Universitaria transformadora (Gaete, 2010; García y Cotrina, 2015; Paéz y Puig, 2013).

Focalizar la formación inicial docente en estrategias de ApS permite a los estudiantes cuestionar los elementos curriculares y organizativos de la Educación Superior (Gutiérrez y Moreno, 2018) y que desarrollen una conciencia social (Puig et al., 2017; Print, 2003). Además de las competencias específicas de la profesión, lo que conduce a repensar la enseñanza como 
actividad compleja, política, ideológica y ética. Al utilizar una metodología de ApS se consigue una auténtica aplicación de los contenidos del currículum a la vida real, puesto que el alumnado conecta con la comunidad, ya que debe emplear sus conocimientos para solventar problemas de su entorno (Ciesielkewicz et al., 2017; Deeley, 2016; Puig, et al., 2007; Vázquez et al., 2017; Mendia, 2013). Así es como consiguen un aprendizaje profundo (Rubio, 2009; Lantieri, 2009; Siegel, 2011) estableciéndose puentes entre el mundo académico y el social (Gil-Gómez, et al., 2016), integrando teoría y práctica, formación y compromiso, cognición y emoción (Manzano, 2010).

Al realizar una revisión de la literatura científica, se evidencia que en los últimos años ha crecido el interés por la puesta en práctica y evaluación de esta metodología, tanto en la enseñanza universitaria (López-Fernández y Benítez-Porres, 2018; Capella-Peris et al., 2020; GómezHurtado et al., 2020; Moliner et al., 2020; Pastor et al., 2020) como en otras etapas educativas (Bär y Puig, 2018; Cervantes et al., 2018; De la Hoz et al., 2018; Ochoa y Pérez, 2019).

En el contexto universitario, Uribe (2018) realizó un estudio cualitativo en el que participaron 26 estudiantes universitarios del Grado de Educación Infantil, para comprobar en qué medida el ApS desarrollaba las competencias propuestas en el currículum. Los resultados mostraron que las percepciones de los participantes hacia la puesta en práctica de dicha metodología habían sido muy positivas, tanto en lo relativo a su desarrollo personal como profesional, destacando el desarrollo de competencias genéricas relacionadas con la ética, diversidad y trabajo en equipo. Por su parte, Fuentes et al. (2020) realizaron una experiencia de ApS con estudiantes del Grado de Educación Primaria de la Universidad Complutense de Madrid junto a un centro educativo de su localidad. Los resultados mostraron que el alumnado participante en el estudio, tras el mismo, habían aumentado su capacidad de reflexión crítica, problematizar la realidad y buscar soluciones, su creatividad y motivación, además una notable adquisición de los conceptos curriculares, pero demandaban más formación en ApS. En la misma línea, hay estudios que afirman que en la Educación Superior este tipo de metodología favorece el desarrollo de competencias emocionales (García y Sánchez, 2017) y competencias interculturales (Losada et al., 2019). Por su parte, Pastor y Muñoz (2019) diseñaron un instrumento de evaluación de proyectos de ApS en la formación inicial docente, al igual que Rodríguez-Izquierdo (2019). También se han evaluado Jornadas divulgativas de ApS (Mayor et al., 2019) con un total de 220 participantes, obteniéndose resultados muy positivos donde se destacó la utilidad del contenido y la importancia de difundir, promover e institucionalizar las prácticas de ApS.

El objetivo del presente estudio es analizar el nivel de satisfacción de los estudiantes del Grado en Educación Infantil en relación a la puesta en práctica de un proyecto de ApS, puesto que la evaluación de dicha estrategia formativa favorece el aprendizaje de los estudiantes (RuizCorbella y García-Gutiérrez, 2019) y permite establecer estrategias de mejora para posteriores cursos académicos.

\section{Descripción de la Experiencia objeto de estudio}

La experiencia mostrada fue puesta en práctica durante el curso 2018/19 en el primer curso del Grado de Maestro/a en Educación Infantil de la Universidad de Málaga, en los grupos A y B de primer curso.

Se puso en práctica una experiencia ApS, cuya finalidad era analizar, organizar, diseñar y ejecutar, desde el punto de vista didáctico, organizativo e inclusivo, un patio más educativo para el 2 o Ciclo de Educación Infantil de un Colegio Público de Infantil y Primaria, de Málaga capital. La pretensión era convertir ese patio de hormigón gris en un entorno de aprendizaje polivalente, flexible, estético e inclusivo, a través de diversos ambientes de aprendizaje. 
Este proyecto debía asegurar que los estudiantes adquirieran y mejorasen las competencias a las que aspira el Grado Maestra/o en Educación Infantil mediante el servicio a dicho centro educativo.

En el Grupo B la propuesta pudo llevarse a cabo desde las asignaturas de "Organización escolar en la Educación Infantil", "Didáctica de la Educación Infantil” y "Hacia una Escuela inclusiva: modelos y prácticas", cuyas profesoras poseían una trayectoria de colaboración previa. Sin embargo, en el Grupo A, estuvieron implicadas tan solo las dos primeras asignaturas, siendo la primera vez que las docentes trabajaban de manera coordinada.

\section{Metodología}

\subsection{Diseño}

El objetivo fundamental de este estudio es valorar el grado de satisfacción de los estudiantes que participaron en el Proyecto de ApS anteriormente descrito. Para ello se realizó un estudio diagnóstico-descriptivo, ex post-facto, desde la complementariedad metodológica cualitativacuantitativa, para así poder comprender las reflexiones y valoraciones del alumnado respecto a la experiencia de ApS.

Las hipótesis de investigación del presente trabajo se han concretado en:

- Existen diferencias significativas respecto a la satisfacción general del alumnado en relación con el Grupo (A o B) de pertenencia ( $p<0.05)$.

- Existen diferencias significativas en relación con el nivel de satisfacción general del alumnado y el haber participado anteriormente en Proyectos de ApS $(p<0.05)$.

- Respecto al estudio cualitativo se ha planteado la siguiente pregunta de investigación: ¿El programa de ApS ha permitido al alumnado desarrollar competencias profesionales, tanto de aprendizajes formales o curriculares como de valores de ciudadanía?

Los datos cuantitativos se han recogido mediante un cuestionario elaborado por las profesoras ad hoc, para conocer el grado de satisfacción y de valoración del impacto del ApS en el alumnado, puesto que es una manera de evaluación de los programas de ApS universitario teniendo en cuenta diferentes dimensiones (Bringle et al., 2004). Se trata de una escala Likert de 5 puntos, en la que el 1 representa "muy en desacuerdo" y el 5 "muy de acuerdo". El instrumento consta de una serie de datos de identificación, y un total de 23 ítems, organizados en 4 bloques: Aportación del ApS a la formación del alumnado; Participación del alumnado y de las profesoras en el diseño y desarrollo didáctico; Compromiso con la propuesta y Valoración general. Dicho instrumento ha sido validado mediante juicio de expertos (3 docentes experimentados en la práctica y la investigación del Aps en la Universidad). La fiabilidad del instrumento fue medida mediante el estadístico Alpha de Cronbach, obteniéndose un valor de ,969, por lo que se puede considerar que dicha fiabilidad es muy alta. Los datos fueron analizados mediante el paquete estadístico SPSS v.21 para Windows.

Los datos cualitativos se han recopilado a partir de las narraciones del alumnado registradas en los Informes de los trabajos grupales y en los Portafolios individuales. El tratamiento de los datos cualitativos se realizó mediante un análisis de contenido categorial, teniendo en cuenta como nodos de referencia las perspectivas propuestas por Butin (2003). En total se han tenido en cuenta 4 perspectivas organizadas en núcleos de contenido, que hacen referencia a los diferentes tipos de aprendizajes que el alumnado adquiere al participar en ApS. Se distinguen: perspectiva técnica (aprendizajes curriculares), cultural (potenciación de la diversidad), política (análisis de la sociedad desde un punto de vista crítico) y aprendizaje postestructural (aprendizajes que desarrollan la construcción de la identidad a partir de las reflexiones respecto 
a la propia experiencia). La información cualitativa proporcionada por el alumnado fue identificada, reducida a nodos de contenido, codificada en sistemas de categorías y analizada para emitir resultados.

\subsection{Participantes}

En total han participado una muestra de 76 alumnas/os, de un total de 136 estudiantes matriculados, realizándose un muestreo de tipo intencional por conglomerados. En relación a los participantes, el 97,4\% eran mujeres, frente a un 2,6\% de hombres, proporción habitual en la titulación del Grado en Educación Infantil. El 53,9\% eran estudiantes matriculados en el Grupo A y el $46,1 \%$ en el Grupo B. Respecto a la edad, el 79,7\% son menores de 20 años, y el $20,3 \%$ oscina en una edad de entre 21 y 24 años. El 90,8\% procede de Bachillerato y el resto ha accedido al Grado mediante una titulación de Técnico Superior.

\section{Resultados}

En el análisis descriptivo del cuestionario se puede apreciar (Tabla 1) que el alumnado valora todos los ítems positivamente, puesto que no existe ninguna media por debajo de 3,30 puntos. A pesar de ello, las valoraciones más positivas son las relacionadas con la capacidad para el trabajo en equipo (ítem v6.4, $\mu=4,47$ ), el incremento de la creatividad del alumnado (ítem v6.8, $\mu=4,42$ ) y la implicación del alumnado en el proyecto para la ejecución del Servicio en el Patio de Infantil (ítem v17.2, $\mu=4,41$ ).

Tabla 1.

Estadísticos descriptivos. Fuente: elaboración propia

\begin{tabular}{l|rrr}
\hline & N & Media & Desv. típ. \\
\hline v1 & 76 & 3,92 & 1,043 \\
v2 & 76 & 3,59 & 1,338 \\
v3 & 76 & 4,03 & 1,143 \\
v4.1 & 76 & 3,79 & 1,181 \\
v4.2 & 76 & 3,58 & 1,289 \\
v4.3 & 75 & 3,91 & 1,232 \\
v4.4 & 76 & 4,20 & 1,020 \\
v4.5 & 76 & 4,17 & 1,063 \\
v4.6 & 76 & 4,13 & 1,050 \\
v4.7 & 74 & 4,12 &, 936 \\
v5 & 68 & 3,71 & 1,120 \\
v6.1 & 76 & 3,39 & 1,690 \\
v6.2 & 76 & 3,36 & 1,140 \\
v6.3 & 76 & 4,00 &, 993 \\
v6.4 & 76 & 4,47 &, 791 \\
v6.5 & 76 & 3,74 & 1,226 \\
v6.6 & 76 & 4,00 & 1,143 \\
v6.7 & 76 & 3,61 & 1,156 \\
v6.8 & 76 & 4,42 &, 753 \\
v6.9 & 75 & 3,51 & 1,132 \\
v7 & 76 & 3,86 & 1,104 \\
v8 & 76 & 3,46 & 1,238 \\
v9 & 76 & 3,55 & 1,076 \\
\hline
\end{tabular}




\begin{tabular}{l|rrr}
\hline & N & Media & Desv. típ. \\
\hline v10 & 76 & 3,51 & 1,052 \\
v11 & 73 & 3,67 & 1,081 \\
v12 & 75 & 3,35 & 1,121 \\
v13 & 76 & 3,54 & 1,051 \\
v14 & 76 & 3,36 & 1,262 \\
v15.1 & 76 & 3,64 & 1,042 \\
v15.2 & 76 & 3,99 & 1,101 \\
v16.1 & 76 & 3,88 & 1,058 \\
v16.2 & 76 & 4,17 &, 885 \\
v17.1 & 76 & 4,14 &, 934 \\
v17.2 & 76 & 4,41 &, 786 \\
v18.1 & 76 & 3,46 & 1,361 \\
v18.2 & 76 & 3,76 & 1,253 \\
v18.3 & 76 & 3,95 & 1,165 \\
v18.4 & 76 & 3,89 & 1,239 \\
v19 & 76 & 4,01 & 1,291 \\
v20 & 76 & 3,93 & 1,268 \\
v21 & 74 & 3,85 & 1,016 \\
N válido (según lista) & 59 & & \\
\hline
\end{tabular}

Este alumnado, en un $71,3 \%$, concluye que su nivel de satisfacción general respecto a la participación en el proyecto de ApS se puede considerar como muy positiva.

Para profundizar más en estos resultados, se ha realizado un contraste de hipótesis mediante pruebas no paramétricas para muestras independientes, ante la imposibilidad de garantizar los supuestos paramétricos de homocedasticidad y normalidad. Se ha empleado la prueba $\mathrm{H}$ de Kruskal-Wallis para analizar la diferencia en la distribución de rangos entre los grupos participantes en el estudio. Se llevó a cabo un primer contraste bilateral para contrastar la igualdad de medias respecto a la satisfacción general del alumnado en el Grupo A y en el Grupo B (Tabla 2).

Tabla 2.

Contraste de hipótesis satisfacción general-grupo de pertenencia. Fuente: elaboración propia

\begin{tabular}{lrrrrr}
\hline v21 & \multicolumn{6}{c}{ Suma de } & gl & \multicolumn{1}{c}{ Media } & F & Sig. \\
& $\begin{array}{c}\text { cuadrática } \\
\text { cuadrados }\end{array}$ & & & \\
Inter-grupos & 22,127 & 1 & 22,127 & 29,925 &, 000 \\
Intra-grupos & 53,238 & 72 &, 739 & & \\
Total & 75,365 & 73 & & & \\
\hline
\end{tabular}

Según la opinión del alumnado existen diferencias entre el grado de satisfacción general entre el Grupo A y B ( $p$-valor=,001) por lo que se rechaza la hipótesis nula. Es decir, pertenecer a un Grupo u otro ha condicionado la satisfacción general respecto al Proyecto de ApS.

Como se puede observar en el gráfico 1, la satisfacción general ha sido considerablemente mayor en el Grupo B que en el Grupo A, existiendo más de un punto de diferencia. 


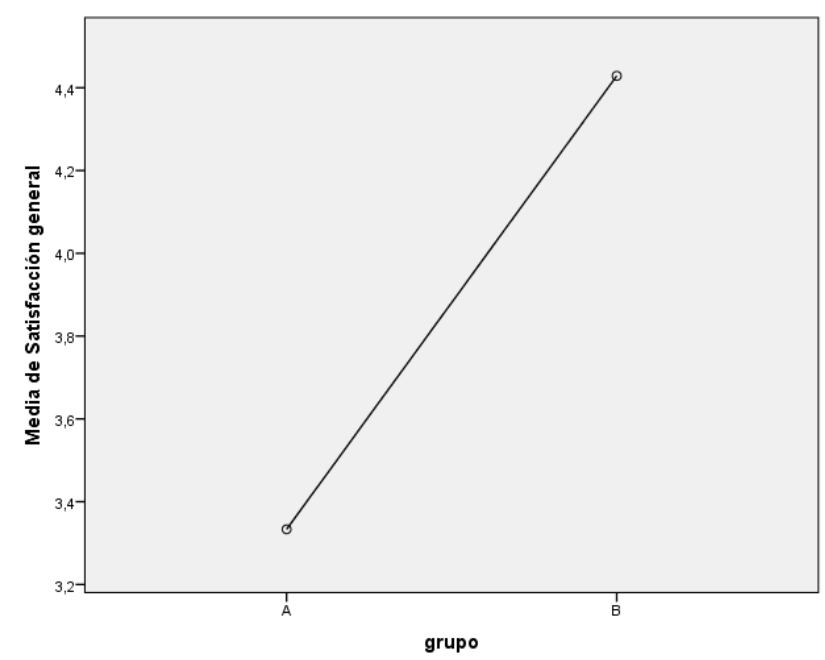

Gráfico 1. Gráfico de medias. Fuente: elaboración propia

En segundo lugar, se ha realizado un contraste de medias para comprobar si los estudiantes que anteriormente han participado en Proyectos de ApS están más satisfechos que aquellos otros que nunca han participado en un proyecto de estas características. A continuación, se muestran los resultados en la Tabla 3.

Tabla 3.

Contraste de hipótesis Satisfacción general - Haber participado con anterioridad en ApS. Fuente: elaboración propia

\begin{tabular}{lrrrrr}
\hline & $\begin{array}{c}\text { Suma de } \\
\text { cuadrados }\end{array}$ & gl & \multicolumn{1}{c}{$\begin{array}{c}\text { Media } \\
\text { cuadrática }\end{array}$} & F & Sig. \\
\hline Inter-grupos &, 479 & 4 &, 120 &, 947 &, 444 \\
Intra-grupos & 7,214 & 57 &, 127 & & \\
Total & 7,694 & 61 & & & \\
\hline
\end{tabular}

Analizados los datos, se obtiene que no existen diferencias significativas ( $p$-valor $=444$ ), lo cual hace que se confirme la hipótesis nula, es decir, que no existen diferencias de medias respecto a la satisfacción general entre los estudiantes que han participado con anterioridad en proyectos de ApS y los que no; consecuentemente, dicha participación no ha condicionado la satisfacción del alumnado respecto a la presente propuesta.

Respecto al análisis cualitativo del contenido de los informes de los trabajos grupales y de los portafolios individuales del alumnado, las evidencias se han organizado en base a las cuatro categorías antes mencionadas: perspectiva técnica, cultural, política y postestructural. De todas ellas la categoría predominante ha sido la dimensión postestructural con un $42,8 \%$ de presencia, seguida de la técnica con un $28,5 \%$ y la política con un $19,2 \%$. La menos evidente ha sido la dimensión cultural (9,5\%). A continuación, se incluyen los resultados más destacados.

La dimensión postestructural hace referencia a que el proyecto de ApS ha permitido a este alumnado desarrollar su identidad, tanto personal como profesional, a través de una experiencia muy enriquecedora: 
Lo cierto es que, como en todo viaje, surgieron complicaciones. Tuvimos la tentación de buscar nuevas rutas, de separar nuestra travesía, de no tener que compartir nada más. Pero decidimos hacer un llamamiento a la madurez, al respeto, a la empatía y al diálogo, y pacíficamente, intentamos seguir en la vía, porque al fin y al cabo queremos educar, queremos enseñar unos valores que, si queremos darlos a conocer, debemos hacerlo con criterio y siendo consecuentes con nuestros actos (Informe del Trabajo grupal no 11, p. 13).

Además, hay una toma de conciencia de la importancia de estar continuamente reciclándose y actualizándose para dar respuestas a las demandas educativas que se les plantean: "considero que una maestra debe estar en continuo aprendizaje siempre" (Portafolios de C.M.J., p. 51).

Respecto a la perspectiva técnica afirman que poner en práctica lo aprendido constituye un aprendizaje relevante:

[...] para nosotras no hay mejor forma de aprender que poniendo en práctica lo aprendido, y gracias a la oportunidad de llevar a la práctica un caso real, hemos conseguido aprender desde la experiencia y desde ahí, nada se olvida" (Informe del Trabajo grupal no 1, p. 17).

Se puede afirmar, así pues, que el ApS, proporciona un aprendizaje profundo en el alumnado. Les ha permitido potenciar la imaginación, han atendido a aspectos del currículum de la Educación Infantil, de la valoración y mejora del grado de inclusión del patio del centro teniendo presentes los indicadores del Index for Inclusion (Booth y Ainscow, 2015) y de los criterios del Diseño Universal para el Aprendizaje (CAST, 2011), para poder realizar un análisis en profundidad del diseño del patio (Informe del Trabajo grupal no 10, p.15).

En definitiva, tal y como afirma M.R.R., el trabajo ha sido enriquecedor y a la vez gratificante:

Asimismo, por mi propia experiencia con nuestro trabajo en el CEIP, puedo confirmarlo y, además, añadir que con el Aprendizaje-Servicio se experimentan situaciones en primera persona mientras se aprende de la forma más activa y participativa posible, consiguiendo potenciar la creatividad y habilidades de todo tipo, adquirir aptitudes y la capacidad de trabajo cooperativo, así como motivar al alumnado y brindarle una mayor autoestima (Portafolios de M.R.R, p. 44).

\section{Discusión y Conclusiones}

En los últimos años se han desarrollado estudios donde se han evidenciado los efectos positivos derivados de la aplicación del ApS en la Educación Superior (Conway et al., 2009; Yorio y Ye, 2012) puesto que participar en este tipo de iniciativas, mejora el aprendizaje respecto al ejercicio profesional (Berríos et al., 2012).

En esta investigación se ha pretendido comprobar el nivel de satisfacción del alumnado en su participación en un Proyecto de ApS, proyecto usado como metodología formativa en la formación inicial de quienes serán maestras/os de Educación Infantil. Se puede concluir que la valoración general del alumnado hacia la puesta en práctica de la metodología de ApS ha sido muy positiva, resultados que coinciden con los obtenidos por Uribe (2018), Fuentes et al. (2020) y Mayor et al. (2019).

Como ya se argumentó en la revisión de la literatura de este estudio, se ha podido comprobar que participar en el Proyecto de ApS ha permitido que el alumnado no sólo aprenda el contenido de las asignaturas, sino que desarrolle competencias profesionales que incluya actitudes de colaboración, valores, responsabilidad social, autonomía, creatividad, etc. Lo cual se corrobora con los resultados obtenidos por diferentes investigaciones realizadas en este ámbito 
(Ciesielkewicz et al., 2017; Deeley, 2016; García y Sánchez, 2017; Losada et al., 2019; Mendia, 2013; Puig, et al., 2007; Vázquez et al., 2017; Videla y Aramburuzabala, 2015; Zayas et al., 2019)

En este caso, los resultados obtenidos, tanto en el análisis cualitativo como en el cuantitativo, del presente estudio, resaltan la utilidad de esta metodología para el desarrollo de la creatividad del alumnado, su implicación en el servicio, así como para potenciar el trabajo en equipo. En esto último es coincidente en con el estudio realizado por Jouannet et al. (2013) donde evaluaron la implementación del ApS en la Universidad Pontificia Católica de Chile, en una muestra de 18.000 estudiantes, obteniéndose como principales resultados que dicha metodología aumentaba la motivación por aprender, el desarrollo de habilidades interpersonales y el trabajo en equipo, y proporcionaba un mayor dominio de competencias técnicas y el desarrollo de valores. Gil-Gómez, et al. (2016) recogen que participar en programas de ApS produce mejoras en la comunicación, en el compañerismo y en la motivación ante el trabajo, coincidiendo estos resultados con los obtenidos por Billig et al. (2005). Del mismo modo, en este trabajo, como se ha indicado anteriormente, se han obtenido valoraciones especialmente positivas respecto al fomento del trabajo en equipo, pero también respecto al desarrollo de la comunicación y fomento de la creatividad.

Por otro lado, en la evaluación cuantitativa se aprecia cierta dejadez en la implicación del alumnado en el diseño progresivo del proyecto, lo cual puede ser debido a que sería necesario antes de empezar un proyecto de ApS proporcionar a dicho alumnado una mayor base teórica respecto al mismo, para que realmente lleguen a entenderlo en profundidad, y a comprender los entresijos del mismo, tanto en su diseño, como en su desarrollo y evaluación.

Además, se aprecia una tendencia de autovaloración del propio trabajo muy positiva, no apreciándose tanto la participación del resto de compañeros/as en los pequeños grupos y, mucho menos, en el gran grupo. Esto puede ser debido a que es necesario reforzar valores sociales como el compañerismo, la solidaridad, la empatía, la autocrítica, etc., elementos que en este estudio se han Ilamado "aprendizajes de utilidad ética".

En este estudio, además, hay que tener presente que los estudiantes que han participado en la experiencia son estudiantes de primer curso de carrera, y esta metodología les ha impulsado a salir de su zona de confort y hacer frente a la incertidumbre y resistencias que les ocasionaba no trabajar mediante una metodología tradicional, puesto que han formado parte de una propuesta interdisciplinar de varias asignaturas, estrategias de aprendizaje-servicio, debían usar por primera vez un portafolios como recurso para la evaluación, etc. Aunque la satisfacción finalmente fue alta o muy alta, el inicio resultó tener un coste emocional ya mencionado en otros estudios (Valero, 2007).

Según Cram (1998) la oportunidad de influir en la mejora de las vidas de otros genera un sentimiento de éxito y el $96 \%$ de los estudiantes que realizaron ApS manifestaron en su estudio estar dispuestos a participar nuevamente. En el presente trabajo, aunque el porcentaje es inferior, concretamente el $67,1 \%$, también estarían dispuestos a participar en futuros Proyectos de ApS. Hay que indicar que, aunque el porcentaje sea ligeramente inferior al obtenido por dicho autor, no deja de ser un resultado positivo.

Así mismo, Hutchins (2012) y Chiva et al. (2018) consideran que los graduados mejoran la confianza en sí mismos y en su sentido del deber ciudadano gracias a su participación en proyectos de ApS. En este estudio se corroboran dichos resultados, en gran medida, debido a que un $80,3 \%$ del alumnado participante considera que formarse mediante Proyectos de ApS les ha permitido aumentar su responsabilidad social.

Respecto a la comparación entre el nivel de satisfacción de los Grupos A y B se puede afirmar que en el Grupo B el alumnado ha mostrado un nivel de satisfacción general notablemente mayor al Grupo A. Y observados los resultados cuantitativos, se puede afirmar que uno de los 
condicionantes de nicho nivel de satisfacción ha podido ser el profesorado, puesto que en el Grupo B han colaborado 3 profesoras con una larga experiencia de trabajo compartido y en el Grupo A, la colaboración ha sido entre 2 docentes que se coordinaban por primera vez. Por esta razón, parece que, para abordar proyectos menos tradicionales de cierta envergadura, el hecho de que el profesorado tenga experiencia previa en trabajos de equipo, puede convertirse en un facilitador del éxito de los mismos.

En el estudio cualitativo se han confirmado los resultados obtenidos por García y Sánchez (2017), puesto que participar en proyectos de ApS ha permitido también en el alumnado desarrollar aspectos emocionales. Además de desarrollar valores que humanizan y que son imprescindibles para el desarrollo social, profesional y personal. Por lo cual, se puede dar una respuesta afirmativa a la pregunta de investigación del presente trabajo, debido a que el Programa de ApS ha permitido al alumnado desarrollar competencias profesionales, tanto de aprendizajes curriculares como de valores de ciudadanía, desarrollando su identidad personal y profesional mediante una experiencia de ApS valorada como enriquecedora.

En definitiva, se puede afirmar que hoy en día es fundamental plantear propuestas de ApS de estas características, las cuales se incardinen en el Plan de Estudios de una manera estructural, para que potencien el desarrollo de actitudes, habilidades y conocimientos imprescindible trabajo en equipo que demanda la sociedad actual. Del mismo modo, si estas experiencias de ApS, u otras menos convencionales, estuvieran más presentes en los Planes de Estudio, el alumnado no viviría estas prácticas como algo aislado o puntual, dentro de una carrera donde la mayoría de las materias siguen con un enfoque metodológico más tradicional, lo que puede que le haga valorar menos la relación coste-beneficio de su participación en propuestas más innovadoras y retadoras (Valero, 2007). Así, se podría ir creando y fortaleciendo una cultura de servicio social en la Educación Superior, que redundaría en la optimización de la calidad y relevancia de los resultados formativos del alumnado universitario y en el mayor calado social de los servicios prestados. Tal y como indican Martínez-Usarralde et al. (2017), habrá que insistir sobre una concepción de Responsabilidad Social Universitaria planteada desde el aula que fomente saberes, prácticas, actitudes y tareas en pro de una ciudadanía responsable que participa en la construcción de una universidad posible y deseable.

La actual sociedad de la información y del conocimiento reclama que el alumnado adquiera herramientas que le capacite para resolver problemas en escenarios de alta complejidad y de renovación constante de los saberes (Jouannet et al., 2013; Simó, 2013). Auqneu, por otro lado, los contextos democráticos actuales, en los que crece progresivamente la desigualdad, exigen que se le oriente igualmente en el desarrollo de los derechos y deberes de su ciudadanía, una ciudadanía participativa y solidaria.

Todo lo que acontece es premisa para concluir que el escenario político, económico y social sin duda ha sufrido importantes cambios, instaurando valores dominantes alejados de los educativos. En este sentido, las instituciones universitarias deben desprenderse de la concepción de individuo sumiso, consumidor, cliente que deja a un lado la política social centrándose en el interés del capitalismo para apostar por propuestas pedagógicas de la construcción y de la creatividad. Este tipo de propuestas permitirán acercar a su alumnado, como ser social, a reivindicar un modelo económico, político y social más justo, humanizado e ilusionante (Carbonell, 2001). Las propuestas de ApS son una estrategia de docencia y aprendizaje acorde a la construcción de este modelo que deberían formar parte de un enfoque político de las universidades.

En este estudio se han encontrado algunas limitaciones. La primera es el tamaño de la muestra que, ampliándola, sería más representativa de la población objeto de estudio, lo que permitiría la generalización de los resultados. Igualmente, también sería interesante realizar un estudio longitudinal del uso del ApS para comprobar su eficacia en el tiempo, puesto que en esta 
investigación sólo se ha realizado el proyecto en un semestre. Como respuesta a algunas de las limitaciones comentadas, las profesoras implicadas han asumido el reto de participar en un Proyecto de Innovación Educativa de la Universidad de Málaga cuyo objetivo está siendo durante este curso 2019/20, establecer procesos de coordinación y aprendizaje compartido entre docentes de la Facultad de Ciencias de la Educación, con práctica o interés en el ApS para, pasando al diseño contrastado, finalmente en los dos próximos cursos, volver a poner en acción nuevos proyectos coordinados en diversas asignaturas de varias de las titulaciones de dicha Facultad.

\section{Referencias bibliográficas}

Angulo, J.F. (1995). El neoliberalismo o el surgimiento del mercado educativo. Kikiriki, cooperación educativa, 35, 25-33.

Aramburuzabala, P., Cerrillo, R. y Tello, I. (2015). Aprendizaje-Servicio: una propuesta metodológica para la introducción de la sostenibilidad curricular en la Universidad. Profesorado. Revista de Currículum y Formación de Profesorado, 19 (1), 78-95.

Bär, B. y Puig, J. M. (2018). Cada curso un aprendizaje-servicio. RIDAS. Revista Iberoamericana de Aprendizaje Servicio, 6, 69-87.

Berríos, V., Contreras, A., Herrada, M., Robles, M.C. y Rubio, X. (2012). Resultados de Aprendizaje Servicio en la UC: informe final de investigación. Recuperado de http://fondedoc.uc.cl/documentos

Billig, S., Root, S. y Jesse, D. (2005). The impact of participation in service-learning on high school students'civic engagement. Circle working paper, 33. Recuperado de http://www.civicyouth.org/PopUps/WorkingPapers/WP33Billig.pdf

Booth, T. y Ainscow, M. (2015). Guía para la inclusión educativa: desarrollando el aprendizaje y la participación en los centros escolares. Madrid: OEI y FUHEM.

Bringle, R., Phillips, M. y Hudson, M. (2004). The measure of service learning. Research scales to assess student experiencies. Washington DC: American Psychological Association.

Butin, D.W. (2003). Of what is it? Multiple conceptualizations of service learning within education. Teachers college record, 105 (9), 1674-1692.

CAST (2011). Universal Design for Learning Guidelines version 2.0. Wakefield, MA: Author Recuperado de http://www.educadua.es/html/dua/ pautasDUA/dua_pautas.html

Capella-Peris, C., Salvador-García, C., Chiva-Bartoll, Ò., y Ruiz-Montero, P. J. (2020). Alcance del aprendizaje-servicio en la formación inicial docente de educación física: una aproximación metodológica mixta. Retos, 37 (37), 465-472.

Carbonell, J. (2001). La aventura de innovar. El cambio en la escuela. Madrid: Morata.

Cervantes, A.O., Galván, L.M.P., y Salinas, J.J. (2018). El aprendizaje-servicio (APS) como práctica expansiva y transformadora. Revista Iberoamericana de Educación, 76, 15-34.

Chiva, O., Pallarés-Piquer, M. y Gil-Gómez, J. (2018). Aprendizaje-servicio y mejora de la Personalidad Eficaz en futuros docentes de Educacije-servic. Revista Complutense de Educación, 29 (1), 181-197. doi: 10.5209/RCED.5216

Cram, S.B. (1998). Impact of service-learning on moral development and self-esteem of community college ethics students. Recuperado de http://eric.ed.gow/?id=ED460701 
CRUE (2015). Institucionalización del Aprendizaje-Servicio como estrategia docente dentro del marco de la Responsabilidad Social Universitaria para la promoción de la Sostenibilidad en la Universidad. Madrid: CRUE Universidades Españolas.

CRUE (2017). Declaración de las universidades españolas a favor del comercio justo y el consumo responsable. Recuperado de: http://www.ocud.es/es/files/doc913/declaracionuniversidades-a-favor-del-comercio-justo-y-el-consumo-responsable.pdf

Ciesielkewicz, M., Nocito, G. y Herrero, Y. (2017). Impacto y beneficio de la metodología de aprendizaje servicio para el profesorado de Educación Superior. Aula de Encuentro, 19 (2), 6-33. doi: 10.17561/ae.v19i2.2

Conde, S., García Prieto, F.J., y Delgado-García, M. (2019). Diseño y validación de un instrumento para analizar el trabajo por rincones en las aulas de Educación Infantil. Estudios sobre Educación, 36, 53-83. doi: 10.15581/004.36.53-83

Conway, J.M., Amel, E.L. y Gerwien, D.P. (2009). Teaching and learning in the social context: a meta-analysis of service learning's effects on academic, personal, social, and citizenship outcomes. Teaching of psychology, 36 (4), 233-245.

De la Hoz, C.J., Fernández, B.A., Olivares, M.I.V. y Hernández, A.B.C. (2018). Volando cometas: proyecto de aprendizaje-servicio entre centros inclusivos. Indivisa: Boletín de estudios e investigación, 18, 65-95.

Deeley, S. (2016). El aprendizaje-Servicio en Educación Superior. Teoría, práctica y perspectiva crítica. Madrid: Narcea.

España. Ley Orgánica 6/2001, de 21 de diciembre, de Universidades. Boletín Oficial del Estado, de 24 de diciembre de 2001, 307, 1-58. Recuperado de: https://www.boe.es/buscar/pdf/2001/BOE-A-2001-24515-consolidado.pdf

España. Real Decreto 1791/2010, de 30 de diciembre, por el que se aprueba el Estatuto del Estudiante Universitario. Boletín Oficial del Estado, de 31 de diciembre de 2010, 318, 109353- 109380. Recuperado de: https://www.boe.es/boe/dias/2010/12/31/pdfs/BOEA-2010-20147.pdf

España. Real Decreto 96/2014, de 14 de febrero, por el que se modifican los Reales Decretos 1027/2011, de 15 de julio, por el que se establece el Marco Español de Cualificaciones para la Educación Superior (MECES), y 1393/2007, de 29 de octubre, por el que se establece la ordenación de las enseñanzas universitarias oficiales. Boletín Oficial del Estado, de 5 de marzo de 2014, 55, 20151-20154. Recuperado de: http://www.boe.es/boe/dias/2014/03/05/pdfs/BOE-A-2014-2359.pdf

España. Ley 45/2015, de 14 de octubre, de Voluntariado. Boletín Oficial del Estado, de 15 de octubre de 2015, 247, 95764- 95784. Recuperado de: https://www.boe.es/boe/dias/2015/10/15/pdfs/BOE-A-2015-11072.pdf

Fuentes, J.L., Martín-Ondarza, P., y Redondo Corcobado, P. (2020). El espacio como lugar para la educación cívica: diseño de un patio escolar mediante un poryecto de AprendizajeServicio. RIED. Revista Iberoamericana de Educación a Distancia, 23(1), 149-167. doi: 10.5944/ried.23.1.24496

Gaete, R. (2010). La responsabilidad social universitaria como desafío para la gestión estratégica de la Educación Superior: el caso de España. Revista de Educación, 355, 109-133.

García, M. y Cotrina, M.J. (2015). El aprendizaje y servicio en la formación inicial del profesorado: de las prácticas educativas críticas a la institucionalización curricular. Profesorado. Revista de currículum y formación del profesorado, 19 (1), 8-25. 
García, E. y Lorente, R. (2014). Grado en maestro en educación primaria: motivaciones y preferencias en la elección de mención. Aula de encuentro, 1 (16), 113-119.

García, M. y Sánchez, L. (2017). El aprendizaje servicio y el desarrollo de las competencias emocionales en la formación inicial del profesorado. Contextos educativos, 20, 127-145.

Gil-Gómez, J., Moliner-García, M.O., Civa-Bartoll, O. y García-López, R. (2016). Una experiencia de aprendizaje-servicio en futuros docentes: desarrollo de la competencia social y ciudadana. Revista Complutense de Educación, 27 (1), 53-73.

Gómez-Hurtado, I., Maya, A.M., y García-Rodríguez, M.P. (2020). Aprendizaje Servicio en la Formación Inicial de Docentes en la Universidad de Huelva. El Proyecto INCLUREC. REICE. Revista Iberoamericana sobre Calidad, Eficacia y Cambio en Educación, 18 (1), 105-123.

Gutiérrez, M. y Moreno, P. (2018). El aprendizaje servicio como metodología para la formación integral de los estudiantes universitarios. Edetania, 53, 185-202.

Hutchins, W. (2012). Groomed for citizenship. Educational Leadership, 69 (7), 70-73.

Igelmo-Zaldívar, J. y Quiroga Uceda, P. (2018). La pedagogía ligera en tiempos hipermodernos: el homeschooling, las escuelas Waldorf y la nueva pedagogía ignaciana. Teoría de la Educación. Revista Interuniversitaria, 30 (1), 758-94. doi: 10.1016/j.jenvp.2015.09.003

Jouannet, C., Salas, M.H. y Contreras, M.A. (2013). Modelo de implementación de aprendizaje servicio $(A+S)$ en la UC. Una experiencia que impacta positivamente en la formación profesional integral. Calidad en la educación, 39, 198-212.

Lantieri, L. (2009). Inteligencia emocional infantil y juvenil. Madrid: Aguilar.

López-Fernández, I., y Benítez-Porres, J. (2018). El aprendizaje servicio en la universidad: una experiencia en el marco de una asignatura del Grado en Educación Primaria. Revista de Docencia Universitaria, 16 (2), 195-210.

Losada, A.S., Santos, M.A., y Álvarez, J.G. (2019). El aprendizaje-servicio como vía para el desarrollo de competencias interculturales en la Universidad. Educatio Siglo XXI, 37(1), 73-90.

Lozano, M., Traver, J.A y Segarra, T. (2018). Responsabilidad educativa para un compromiso ciudadano crítico. Archivos Analíticos de Políticas Educativas, 26 (97), 1-19. doi: 10.14507/epaa.26.3688

Manzano, V. (2010). El ApS y su potencial para la educación superior. VI Jornadas de educación en Psicología. Sevilla: Universidad de Sevilla.

Martínez, M. (2006). Formación para la ciudadanía y educación superior. Revista Iberoamericana de Educación, 42, 85-102.

Martínez, B., Martínez, I., Alonso, I. y Gezuraga, M. (2013). El aprendizaje servicio, una oportunidad para avanzar en la innovación educativa dentro de la universidad del País Vasco. Tendencias Pedagógicas, 21, 99-117.

Martínez-Usarralde, M.J., Lloret-Catlá, C. y Mas-Gil. S. (2017). Responsabilidad Social Universitaria (RSU). Principios para una universidad sostenible, cooperativa y democrática desde el diagnóstico participativo de su alumnado. Archivos Analíticos de Políticas Educativas, 25 (75), 1-25. doi: 10.14507/epaa.25.2769

Martínez-Vivot, M. y Folgueiras, P. (2015). Evaluación participativa, aprendizaje-servicio y universidad. Profesorado. Revista de Currículum y Formación de Profesorado, 19 (1), 128143. 
Mayor, D. (2020). El Aprendizaje-Servicio en el ámbito universitario. En D. Mayor, M. Fernández, M. y M.P. Andrés (Eds). Teoría y práctica del Aprendizaje-Servicio en la Universidad (pp. 53-74). Almería: EDUAL.

Mayor, D. y Rodríguez, D. (2017). Aprendizaje-Servicio: una práctica pedagógica que promueve la participación del estudiantado para la mejora escolar y social. Revista Complutense de Educación, 28 (2), 555-571.

Mayor Paredes, D., López Medialdea, A.M. y Solís Galán, M.G. (2019). El Aprendizaje-Servicio como Escenario Formativo y su Influencia en Distintos Agentes Socioeducativos. Percepción de los Participantes. International Journal of Sociology of Education, 8(2), 153172. doi: $10.17583 /$ rise.2019.4071

Mendia, R. (2013). Aprendizaje y servicio solidario: el acompañamiento educativo. Bilbao: Zerbikas.

Moliner García, O., Arnaiz Sánchez, P. y Sanahuja Ribés, A. (2020). Rompiendo la brecha entre teoría y práctica: ¿qué estrategias utilizar el profesorado universitario para movilizar el conocimiento sobre educación inclusiva? Educacion XX1,23(1), 173-195. doi: 10.5944/educXX1.23753.

Ochoa, A. y Pérez, L. (2019). El aprendizaje servicio, una estrategia para impulsar la participación y mejorar la convivencia escolar. Psicoperspectivas, 18 (1), 1-13. http://dx.doi.org/10.5027/psicoperspectivas-vol18-issue1-fulltext-1478

ONU (2019). Objetivos de desarrollo sostenible. Recuperado de: http://www.onu.org.mx/agenda-2030/objetivos-del-desarrollo-sostenible/

Opazo, H., Ramírez, C., García-Peinado, R. y Lorite, M. (2015). Analizar, repensar y mejorar los proyectos: una rúbrica para la autoevaluación de experiencias de Aprendizaje Servicio. Profesorado. Revista de Currículum y Formación del Profesorado, 19 (1), 144-175.

Páez, M. y Puig, J.M. (2013). La reflexión en el aprendizaje-servicio. Revista internacional de educación para la justicia social, 2 (2), 13-32.

Pastor, M.L.S. y Muñoz, L.F.M. (2019). La evaluación formativa y compartida en AprendizajeServicio. Un ejemplo en formación inicial del profesorado. Revista Infancia, Educación y Aprendizaje, 5(2), 595-600.

Pastor, M.L.S., Cañadas, L. y Muñoz, L.F.M. (2020). Limitaciones del aprendizaje-servicio en la formación inicial en actividad físico-deportiva. Retos, 37(37), 509-517.

Print, M. (2003). Estrategias de enseñanza para la educación cívica y ciudadana en el siglo XXI. Estudios sobre Educación, 4, 7-22.

Puig, J.M., Batlle, R., Bosch, C. y Palos, J. (2007). Aprendizaje Servicio: educar para la ciudadanía. Barcelona: Octaedro.

Puig, J.M., Martín, X. y Rubio, L. (2017). ¿Cómo evaluar proyectos de aprendizaje servicio? Voces de la Educación, 2 (2), 122-132.

Rizvi, F. (2010). La educación a lo largo de la vida: más allá del imaginario neo-liberal. Revista Española de Educación Comparada, 16, 185-210.

Rodicio, M.L. (2010). La docencia universitaria en el proceso de adaptación a Europa. La percepción de los estudiantes. Enseñanza \& Teaching: Revista interuniversitaria de didáctica, 28 (2), 23-43. 
Rodríguez-Izquierdo, R.M. (2019). Validación de una escala de medida del impacto del aprendizaje-servicio en el desarrollo de las competencias profesionales de los estudiantes en formación docente. Revista Mexicana de Psicología, 36 (1), 63-73.

Rubio, L. (2009). El aprendizaje en el aprendizaje servicio. En J.M. Puig (coord.). Aprendizaje Servico (ApS). Educación y compromiso cívico (pp. 91-105). Barcelona: Editorial Grao.

Ruiz-Corbella, M., y García-Gutiérrez, J. (2019). Aprendizaje-Servicio. Los retos de la evaluación. Madrid: Narcea.

Santos, M.A. (2016) (Ed.). Sociedad del conocimiento. Aprendizaje e innovación en la universidad. Madrid: Biblioteca Nueva.

Santos, M.A., Jover, G., Naval, C., Álvarez, J.L., Vázquez, V. y Sotelino, A. (2017). Diseño y validación de un cuestionario sobre práctica docente y actitud del profesorado universitario hacia la innovación (CUPAIN). Educación XXI, 20 (2), 39-71.

Siegel, D.J. (2011). Midsight. La nueva ciencia de la transformación personal. Barcelona: Paidós.

Simó, S. (2013). El aprendizaje servicio universitario: materializando el compromiso social de la universidad desde una educación basada en la excelencia. Estudios sobre el Mensaje Periodístico, 19, 1027-1036.

Unesco. (2015). Replantear la educación ¿Hacia un bien común mundial? París: Unesco.

Uribe. P. A. (2018). Percepción de los estudiantes de educación inicial frente al desarrollo de experiencias formativas en modalidad A+S. Revista Electrónica de Investigación Educativa, 20(4), 110-122. doi: 10.24320/redie.2018.20.4.1826

Valero, M. (2007). Las dificultades que tienes cuando haces PBL. En J.A. Marín (Ed.), La Educación Superior hacia la Convergencia Europea: Modelos basados en el aprendizaje. Guipúzcoa: Universidad de Mondragón. Recuperado de http://personals.ac.upc.edu/miguel/materiales/docencia/articulos/dificultades PBL.pdf

Vallaeys, F. (2008). Responsabilidad Social Universitaria: una nueva filosofía de gestión ética e inteligente para las universidades. Revista Educación Superior y Sociedad, 13(2), 191-220.

Vázquez, S., Liesa, M. y Lozano, A. (2017). Recreos Cooperativos e Inclusivos a través de la metodología de Aprendizaje-Servicio. Revista Electrónica Interuniversitaria de Formación del Profesorado, 20(1), 173-185.

Videla, W. y Aramburuzabala, P. (2015). Las Motivaciones de los estudiantes del Grado de Maestro en Educación Infantil para realizar ApS. En P. Aramburuzabala, H. Opazo y J. García-Gutiérrez (ed.), El Aprendizaje-servicio en las universidades. De la iniciativa individual al apoyo institucional. Madrid: UNED. Recuperado de: http://espacio.uned.es/fez/eserv/bibliuned:EditorialUNED-aa-EDU-Jgarcia0001/Garcia_Gutierrez_Juan_aprendizajeservicio.pdf

Yorio, P. L. y Ye, F. (2012). A Meta-Analysis on the Effects of Service-Learning on the Social, Personal, and Cognitive Outcomes of Learning. Academy of Management Learning and Education, 11(1), 9-27.

Zabalza, M.A. (2006). La convergencia como oportunidad para la mejora de la docencia universitaria. Revista Interuniversitaria de Formación del profesorado, 20 (3), 37-69.

Zayas Latorre, B., Gozálvez Pérez, V. y Gracia Calandín, J. (2019). La Dimensión Ética y Ciudadana del Aprendizaje Servicio: Una apuesta por su institucionalización en la Educación Superior. Revista Complutense de Educación, 30 (1), 1-15. Recuperado de: https://revistas.ucm.es/index.php/RCED/article/download/55443/4564456548995 